\title{
CONSUMO DE ADOÇANTES E DE PRODUTOS DIET E LIGHT POR DIABÉTICOS ATENDIDOS EM DUAS UNIDADES BÁSICAS DE SAÚDE DE ALFENAS-MG
}

\author{
Bianca Danielli Goulart ALVES ${ }^{1}$ \\ Mayara Travagli $\operatorname{RAMOS}^{1}$ (in memoriam) \\ Yara Rafaela PAVANI ${ }^{1}$ \\ Tania Mara Rodrigues SIMÕES ${ }^{2 *}$
}

\author{
${ }^{1}$ Acadêmica do curso de Nutrição, Faculdade de Nutrição, Universidade Federal de Alfenas - UNIFAL-MG \\ E-mail: biancagoulart03@yahoo.com.br; yara.pavani@ hotmail.com \\ ${ }^{2}$ Docente da Faculdade de Nutrição, UNIFAL-MG. E-mail: tania.simoes@unifal-mg.edu.br \\ *Autor para correspondência
}

Recebido em: 26/03/2015 - Aprovado em: 15/08/2015 - Disponibilizado em: 30/10/2015

\begin{abstract}
RESUMO
Para o controle metabólico do diabetes, é imprescindível a mudança dos hábitos alimentares. Uma boa alternativa para diminuir as restrições na dieta dos diabéticos é estimular o consumo consciente de produtos "diet" e "light", assim como o de edulcorantes. Objetivou-se caracterizar o consumo de adoçantes e de produtos "diet" e "light" por diabéticos, atendidos em duas Unidades Básicas de Saúde de Alfenas (MG), assim como classificar o estado nutricional (pelo índice de massa corporal) e o risco coronariano desses indivíduos (pela razão cintura/estatura). Foi realizado um estudo transversal, quantitativo e descritivo, com 129 pacientes diabéticos, maiores de 18 anos, que participaram voluntariamente. Os dados pessoais, sociodemográficos, clínicos, antropométricos, de consumo e conhecimento sobre tais alimentos foram coletados com aplicação de um questionário. Foram entrevistadas 81 mulheres $(62,8 \%)$ e 48 homens $(37,2 \%)$. Independentemente de gênero e grupo etário, mais de $60 \%$ dos indivíduos apresentaram excesso de peso e risco coronariano elevado; mais de $70 \%$ dos entrevistados relataram o hábito de consumir edulcorantes e o mesmo percentual relatou não saber a diferença entre produtos "diet" e "light", apesar de 46,5\% afirmarem o consumo desses produtos. Portanto, é de extrema importância que os pacientes diabéticos atendidos pelos serviços de saúde recebam orientações nutricionais sobre o uso correto desses produtos, para uma escolha mais consciente dos mesmos, assim como tenham uma rotina de acompanhamento também com nutricionistas, para prevenção de eventos cardiovasculares graves e melhora da qualidade de vida.

Palavras-chave: Diabetes mellitus. Diet. Light. Edulcorantes. Consumo.
\end{abstract}

\begin{abstract}
For the metabolic control of diabetes, it is essential to change dietary habits. A good alternative to relax restrictions in the diet of diabetics is to stimulate consumer awareness of products "diet" and "light", as well as sweeteners. This study aimed to characterize the consumption of sweeteners and products "diet" and "light" for diabetics, attended by diabetics treated at two Basic Health Units Alfenas (MG), as well as nutritional status classification (by body mass index) and the coronary risk of these individuals (for waist / height). A cross-sectional, quantitative and descriptive study was conducted with 129 diabetic patients aged over 18 years who participated voluntarily. Personal data, sociodemographic, clinical, anthropometric, and consumer knowledge about such foods were collected using a questionnaire. We interviewed 81 women (62.8\%) and 48 men (37.2\%). Regardless of gender and age group, over $60 \%$ of subjects were overweight and high coronary risk; more than $70 \%$ of respondents reported the habit of consuming sweeteners and the same percentage reported not knowing the difference between products "diet" and "light" despite $46.5 \%$ claiming the consumption of these products. Therefore, it is extremely important that diabetic patients treated by health services receive nutritional guidelines on the correct use of these products, for a more conscious choice of the same, as well as have a routine follow-up with nutritionists, to prevent major cardiovascular events and improved quality of life.
\end{abstract}

Keywords: Diabetes mellitus. Diet. Light. Sweeteners. Consumption.

\section{INTRODUÇÃO}

O diabetes mellitus (DM) é uma

patologia de múltiplas causas, decorrente da falta de insulina (DM tipo 1) ou da incapacidade desta de exercer adequadamente suas funções acarretando uma resistência à 
ação deste hormônio (DM tipo 2). Esta patologia causa transtornos no metabolismo intermediário, levando a uma hiperglicemia (principal característica diagnóstica da doença) (PONTIERI; BACHION, 2010).

Para o tratamento do DM é fundamental a mudança dos hábitos alimentares, e o acompanhamento com um profissional da nutrição é imprescindível para o controle metabólico dos pacientes. A terapia nutricional tem o objetivo de auxiliar o paciente a controlar os níveis glicêmicos, o perfil lipídico, a pressão arterial, promovendo uma alimentação saudável e sensibilizando o indivíduo sobre a necessidade de selecionar cada alimento, além de orientá-lo para uma perda de peso saudável e prevenção do ganho ponderal excessivo, com intuito de reduzir a resistência à insulina (BATISTA et al., 2006).

Uma boa alternativa para diminuir as restrições na dieta dos diabéticos é estimular o consumo de produtos "diet" e "light", que vem evoluindo cada vez mais, assim como o de edulcorantes. Os alimentos "diet" são aqueles formulados para indivíduos que apresentam alguma necessidade especial em relação aos aspectos fisiológicos, metabólicos e patológicos, sendo isentos de algum nutriente (BRASIL, 1998; DEGÁSPARI; MOTTIN; RORATO, 2006). De acordo com esses mesmos autores e Brasil (2012), os alimentos "light" são aqueles que sofrem uma redução mínima de $25 \%$ do valor calórico original ou de alguns nutrientes.

Edulcorantes são substâncias com poder adoçante superior ao da sacarose, utilizados como substitutos do açúcar. São classificados em naturais e sintéticos, sendo os primeiros obtidos sem reações químicas. Já os sintéticos são produzidos através de reações químicas podendo ser ou não de produtos naturais (VIGGIANO, 2003).

Tendo em vista a importância do consumo desses produtos para os indivíduos diabéticos, o presente estudo teve o objetivo de caracterizar o consumo de adoçantes (edulcorantes) e de produtos "diet" e "light" por pacientes diabéticos, atendidos pelo Sistema Único de Saúde (SUS) do município de Alfenas (MG), assim como avaliar o estado nutricional e o risco coronariano desses indivíduos.

\section{METODOLOGIA}

Foi realizado um estudo transversal, quantitativo e descritivo, com indivíduos diabéticos maiores de 18 anos, de ambos os gêneros, em duas Unidades Básicas de Saúde (UBS), do município de Alfenas-MG, no período de setembro a outubro de 2014.

Em ambos os locais, nos dias de coleta de dados, inicialmente foram analisados os prontuários dos pacientes agendados, para se verificar os critérios de inclusão (ter diagnóstico médico de DM e idade acima de 
18 anos). Em seguida, era feito o convite para participação voluntária no estudo, explicandose os procedimentos e recolhendo-se a assinatura do termo de consentimento livre e esclarecido, sendo então realizada a entrevista e a avaliação antropométrica. Considerou-se como critérios de exclusão ser menor de idade, não constar o diagnóstico médico de DM no prontuário ou o fato da pessoa não aceitar participar do estudo.

O questionário utilizado abrangeu dados sociodemográficos, clínicos e hábitos pessoais, incluindo o consumo de produtos "diet" e "light" e de edulcorantes, assim como se o usuário sabia identificar a diferença entre tais produtos. Também foi realizada avaliação nutricional antropométrica, com aferição de peso, altura e circunferência da cintura segundo SISVAN (BRASIL, 2008) e classificação de índice de massa corporal (IMC) de adultos e idosos, de acordo com a Organização Mundial de Saúde (WHO, 2000) e Lipschitz (1994), respectivamente, considerando-se os pontos de corte para baixo peso, eutrofia e excesso de peso. Foi calculada e analisada a razão cintura/estatura, considerando-se como risco coronariano elevado quando esta razão foi maior que 0,50 para homens e mulheres (HAUN; PITANGA; LESSA, 2009; PITANGA, 2011).

Ao final da entrevista, foi entregue um folheto educativo, com conceitos de edulcorantes e de produtos "diet" e "light", incluindo orientações nutricionais gerais para DM.

Os dados obtidos foram codificados no software Excel 2010, sendo realizada análise descritiva das variáveis (média, mínimo e máximo, distribuição percentual). $\mathrm{O}$ estudo foi aprovado pelo Comitê de Ética em Pesquisa da UNIFAL-MG (número do parecer: 778.582, de 29/08/2014).

\section{RESULTADOS}

Foram entrevistados 129 indivíduos diabéticos, sendo 62,8\% (n=81) mulheres e $37,2 \%(n=48)$ homens.

A idade média dos entrevistados foi de 58,9 anos, variando de 18 a 86 anos. A distribuição da amostra por grupos etários foi de 5,4\% de indivíduos com 18 a 30 anos, $16,2 \%$ com 31 a 50 anos, $44,2 \%$ com 51 a 65 anos e 34,2\% com mais de 65 anos de idade.

Em relação ao tempo que os indivíduos descobriram o diabetes, 6,2\% sabiam da doença há menos de 1 ano, 17,83\% descobriram a doença de 1 a 3 anos, 13,17\% de 3 a 5 anos, $17,1 \%$ de 5 a 10 anos e a maioria dos indivíduos $(47,7 \%)$ descobriu a doença há mais de 10 anos.

Do total da amostra, $7 \quad(5,4 \%)$ indivíduos apresentaram baixo peso, 41 $(31,8 \%)$ apresentaram eutrofia e $81(62,8 \%)$ apresentaram excesso de peso. Também é mostrada a classificação do IMC dos 
indivíduos agrupados por faixa etária e por gênero (Tabela 1).

Quanto à renda familiar, aproximadamente $70 \%$ da amostra relatou receber de 1 a 3 salários mínimos.
Em relação ao tabagismo e etilismo, $7,8 \%$ e $10,9 \%$ dos entrevistados, respectivamente, declararam apresentar esses hábitos. Quando se questionou se havia alguma doença associada ao DM, as mais citadas foram hipertensão arterial, hipotireoidismo e hipercolesterolemia.

Tabela 1 - Distribuição (\%) da classificação do IMC dos indivíduos diabéticos, segundo faixa etária e gênero. Alfenas (MG), 2014.

\begin{tabular}{|c|c|c|c|c|c|c|}
\hline & \multicolumn{4}{|c|}{ Faixa etária (anos) } & \multicolumn{2}{|c|}{ Gênero } \\
\hline & 18 a 30 & 31 a 50 & 51 a 65 & Acima de 65 & Feminino & Masculino \\
\hline Baixo Peso & $28,6 \%$ & $0 \%$ & $3,5 \%$ & $6,8 \%$ & $6,1 \%$ & $4,1 \%$ \\
\hline Eutrofia & $42,8 \%$ & $19 \%$ & $22,8 \%$ & $47,7 \%$ & $30,9 \%$ & $33,4 \%$ \\
\hline $\begin{array}{l}\text { Excesso de } \\
\text { Peso }\end{array}$ & $28,6 \%$ & $81 \%$ & $73,7 \%$ & $45,5 \%$ & $63 \%$ & $62,5 \%$ \\
\hline
\end{tabular}

FONTE: Dados da pesquisa.

Tanto homens $(85,4 \%$ deles $)$ quanto mulheres $(93,8 \%$ delas) apresentaram risco coronariano elevado, de acordo com a razão cintura/estatura (HAUN; PITANGA; LESSA, 2009). Em relação à idade, dentre aqueles maiores de 65 anos, 95,5\% apresentaram risco coronariano elevado. Dentre os mais jovens, $57,1 \%$ apresentaram tal risco.

Em relação ao uso de edulcorantes, a maioria das mulheres $(72,8 \%)$ respondeu fazer uso desses produtos, sendo que 25,9\% dessas faziam uso três vezes ou mais ao dia. Entre os homens, 77,1\% usavam adoçantes, com 37,5\% deles apresentando também um consumo de três vezes ou mais/dia. Quando questionados sobre como escolhiam seu adoçante, a resposta de maior frequência, independente de gênero e idade, foi o sabor $(44,2 \%)$.

Quanto ao consumo e conhecimento acerca dos produtos "diet" e "light", foi observado que $46,5 \%$ da população entrevistada $(n=60)$ afirmaram o consumo desses alimentos, apesar de apenas 25,6\% dos indivíduos entrevistados $(\mathrm{n}=33)$ declararem saber a diferença entre eles. Disseram não saber a diferença entre esses produtos, $74,4 \%$ (n=96) dos indivíduos avaliados. 
No entanto, quando se questionou "O que é para o(a) $\operatorname{Sr}(\mathrm{a})$. um alimento diet?" (Tabela 2) e "O que é para o(a) $\operatorname{Sr}(a)$. um alimento light?" (Tabela 3), notou-se que a grande maioria dos entrevistados não soube responder às questões sobre o significado desses produtos, independentemente de gênero e idade.

Tabela 2 - Distribuição (\%) das respostas dos indivíduos sobre alimentos "diet", segundo faixa etária e gênero. Alfenas, 2014.

"O que é para o(a) senhor(a) um alimento diet?"

\begin{tabular}{|c|c|c|c|c|c|c|}
\hline \multirow[b]{2}{*}{ Resposta } & \multicolumn{4}{|c|}{ Faixa etária (anos) } & \multicolumn{2}{|c|}{ Gênero } \\
\hline & 18 a 30 & 31 a 50 & 51 a 65 & $\underset{65}{\text { Acima de }}$ & Feminino & Masculino \\
\hline $\begin{array}{l}\text { Que são } \\
\text { isentos de } \\
\text { algum } \\
\text { nutriente } \\
\text { (açúcar, } \\
\text { sódio) }\end{array}$ & $28,6 \%$ & $19 \%$ & $17,5 \%$ & $9,1 \%$ & $18,5 \%$ & $10,4 \%$ \\
\hline $\begin{array}{l}\text { Que engorda } \\
\text { menos }\end{array}$ & $0 \%$ & $0 \%$ & $1,7 \%$ & $0 \%$ & $1,2 \%$ & $0 \%$ \\
\hline $\begin{array}{c}\text { Que é } \\
\text { indicado para } \\
\text { diabéticos }\end{array}$ & $0 \%$ & $4,8 \%$ & $5,4 \%$ & $4,5 \%$ & $7,4 \%$ & $4,2 \%$ \\
\hline $\begin{array}{l}\text { Que não pode } \\
\text { ser } \\
\text { consumido } \\
\text { por diabéticos }\end{array}$ & $28,6 \%$ & $0 \%$ & $3,5 \%$ & $0 \%$ & $2,4 \%$ & $0 \%$ \\
\hline Não sabe & $42,8 \%$ & $76,2 \%$ & $71,9 \%$ & $86,4 \%$ & $70,5 \%$ & $85,4 \%$ \\
\hline
\end{tabular}

FONTE: Dados da pesquisa.

Por outro lado, quando se questionou “ $\mathrm{O}$ (a) $\mathrm{Sr}(\mathrm{a})$. acha que um diabético necessita utilizar alimentos diet e light?", 27\% disseram que somente o uso de alimentos "diet" seria necessário e $26 \%$ da amostra relatou não achar necessário o consumo de tais produtos. A minoria dos entrevistados (5\%) relatou que somente o uso de produtos "light" era necessário e 20,5\% não souberam responder.

Dentre os entrevistados, $90,7 \%$ disseram considerar que a dieta é um cuidado importante no tratamento do diabetes. A maioria $(70,1 \%)$ afirmou já ter recebido tais orientações, seja por médico $(63,7 \%)$, nutricionista $(34,3 \%)$ ou enfermeiro $(2 \%)$. Contudo, 29,9\% afirmaram nunca terem recebido orientações de dieta para diabetes. 
Tabela 3 - Distribuição (\%) das respostas dos indivíduos sobre alimentos "light", segundo faixa etária e gênero. Alfenas, 2014.

"O que é para o(a) senhor(a) um alimento light?"

\begin{tabular}{|c|c|c|c|c|c|c|}
\hline \multirow[b]{2}{*}{ Resposta } & \multicolumn{4}{|c|}{ Faixa etária (anos) } & \multicolumn{2}{|c|}{ Gênero } \\
\hline & 18 a 30 & 31 a 50 & 51 a 65 & $\begin{array}{c}\text { Acima de } \\
65\end{array}$ & Feminino & Masculino \\
\hline $\begin{array}{c}\text { Que tem } \\
\text { redução } \\
\text { mínima de } \\
25 \% \text { de algum } \\
\text { nutriente, } \\
\text { como açúcar } \\
\text { ou sódio }\end{array}$ & $28,6 \%$ & $19,0 \%$ & $12,3 \%$ & $4,5 \%$ & $11,1 \%$ & $10,4 \%$ \\
\hline $\begin{array}{l}\text { Que é indicado } \\
\text { para dietas de } \\
\text { emagrecimento }\end{array}$ & $28,6 \%$ & $9,5 \%$ & $3,5 \%$ & $0 \%$ & $7,4 \%$ & $0 \%$ \\
\hline $\begin{array}{l}\text { Que é indicado } \\
\text { para diabéticos }\end{array}$ & $0 \%$ & $0 \%$ & $1,75 \%$ & $4,5 \%$ & $2,5 \%$ & $2,1 \%$ \\
\hline $\begin{array}{l}\text { Que não pode } \\
\text { ser consumido } \\
\text { por diabéticos }\end{array}$ & $0 \%$ & $0 \%$ & $1,75 \%$ & $0 \%$ & $1,2 \%$ & $0 \%$ \\
\hline Não sabe & $42,8 \%$ & $71,5 \%$ & $80,7 \%$ & $91,0 \%$ & $77,8 \%$ & $87,5 \%$ \\
\hline
\end{tabular}

FONTE: Dados da pesquisa.

\section{DISCUSSÃO}

O IMC é um dos métodos antropométricos mais utilizados para identificar alterações no estado nutricional de adultos e idosos (ABRANTES et al., 2006). Vários estudos têm apontado a razão cintura/estatura como um bom discriminador de obesidade abdominal relacionado a fatores de risco cardiovascular (HAUN; PITANGA; LESSA; 2009; SOUSA et al., 2011). Foi visto em outros estudos a importância de se usar a razão cintura/estatura como discriminador de obesidade central, porque ele minimiza a avaliação errônea de risco cardiovascular em indivíduos adultos de estatura, sexo, etnia, faixa etária diferentes (DUCA et al., 2011).

Quando analisada a classificação do IMC por sexo e faixa etária, observou-se que mais de $60 \%$ da população estudada, de ambos os gêneros, apresentaram excesso de peso, e que a população a partir dos 31 anos 
de idade foi a que apresentou maior índice de excesso de peso. No entanto, cerca de $30 \%$ dos indivíduos jovens avaliados (entre 18 e 30 anos) já estavam com sobrepeso ou obesidade.

Em relação à razão cintura/estatura, observou-se que a maioria da população feminina quanto da masculina e que mais de $80 \%$ dos diabéticos com idade a partir de 31 anos apresentaram risco para doenças cardiovasculares, corroborando os achados de Benedetti e colaboradores (2012), onde $81,4 \%$ dos idosos entrevistados apresentaram razão cintura/estatura inadequada.

Tais dados são bastante preocupantes, sobretudo pelo fato da população em questão ser diabética. Sabe-se que o excesso de peso, principalmente a obesidade central, pode ser um agravante à situação metabólica desses indivíduos, além de aumentar a predisposição à hipertensão arterial e dislipidemias. Por isso, destaca-se a importância da participação de nutricionistas na equipe multidisciplinar nas Unidades Básicas de Saúde visando a um melhor controle do peso corporal e metabólico de diabéticos, para a prevenção de várias complicações, principalmente eventos cardiovasculares graves, como infarto agudo do miocárdio e acidente vascular cerebral (FERREIRA; FERREIRA, 2009).

Quanto ao uso de adoçantes, este foi razoavelmente maior entre os homens do que entre as mulheres, diferenciando-se do estudo feito por Zanini e colaboradores (2011), em Pelotas-RS, que encontrou um consumo de edulcorantes maior no gênero feminino. Em nosso estudo, observou-se uma tendência maior do uso de adoçantes conforme o aumento da idade, o que também foi observado no estudo feito em Pelotas. Contudo, os idosos acima de 65 anos foram os que menos referiram o uso de adoçantes.

Dos entrevistados, mais de $70 \%$ disseram não saber a diferença entre alimentos "diet" e "light" e, mesmo entre aqueles que afirmaram tal conhecimento, notou-se que uma parcela conceituou os produtos de maneira inapropriada. Esses resultados mostram a necessidade de se desenvolver atividades educativas nas unidades de saúde para disseminar tais informações, de modo que os diabéticos possam consumir tais produtos de maneira consciente, de acordo com suas necessidades e patologias (OLIVEIRA; FRANCO, 2010).

Quando os indivíduos foram questionados sobre a necessidade do consumo de produtos "diet" e "light" por diabéticos, grande parte não soube responder. Acredita-se que esse fato se deva à falta de orientação sobre esses produtos por parte da população em geral, não só diabéticos. Apesar de os produtos "diet" serem considerados alimentos para fins especiais, estes costumam ser mais consumidos por indivíduos com objetivos somente estéticos. Informações sobre 
alimentos "diet" e "light" são de bastante utilidade nos serviços de saúde que atendem diabéticos, pois podem contribuir nas escolhas alimentares desses indivíduos propiciando uma maior flexibilidade dietética, devendo-se, no entanto, levar em consideração as características socioeducacionais da população na hora de se passar as orientações (OLIVEIRA; FRANCO, 2010).

A maioria dos homens e mulheres referiu ser importante a dieta para o tratamento do DM. Mais de 70\% dos entrevistados responderam já ter recebido orientação sobre dieta para DM, corroborando os resultados encontrados por Oliveira e Franco (2010), em que mais de 95\% dos indivíduos disseram já ter recebido algum tipo de orientação quanto à alimentação julgando isso importante para o tratamento, apesar de grande parte não seguir tais recomendações.

Em nosso estudo, dentre os indivíduos que afirmaram ter recebido orientações dietéticas para diabetes, menos de $35 \%$ deles as recebeu de nutricionistas, o que nos aponta uma necessidade de ampliação da atenção nutricional no município de Alfenas.

Podemos citar como limitação de nosso estudo, a não realização de avaliação nutricional em relação à ingestão alimentar dos voluntários, o que não permitiu uma análise do consumo de alimentos "diet", "light" e edulcorantes no contexto da alimentação habitual dos diabéticos entrevistados, assim como a investigação sobre realização de atividade física, sugerindo-se, então, essas análises em estudos posteriores.

\section{CONCLUSÃO}

Conclui-se que a maioria dos diabéticos avaliados apresentou excesso de peso e risco elevado para doenças cardiovasculares. A grande maioria deles relatou fazer uso de edulcorantes e quase metade afirmou consumir alimentos "diet" e "light", no entanto, uma expressiva parcela da população entrevistada disse não saber a diferença entre tais produtos. Considera-se de extrema importância que os pacientes diabéticos atendidos pelas duas unidades de saúde recebam orientações nutricionais sobre o uso correto de produtos "diet", "light" e adoçantes, para uma escolha mais consciente dos mesmos e uso em preparações alimentares, para melhor controle da glicemia, facilitando a adesão à dieta para DM.

Faz-se necessário estabelecer uma rotina de acompanhamento dos diabéticos também com nutricionistas, não só com médicos e enfermeiros, o que poderia contribuir para controle de peso corporal, prevenção de eventos cardiovasculares graves e melhora da qualidade de vida. Sendo assim, sugere-se disponibilizar aos diabéticos do município de Alfenas maior acesso à atenção 
nutricional como parte de uma assistência integral à saúde.

\section{REFERÊNCIAS}

ABRANTES et al. Estado nutricional e fatores associados em idosos. Rev Assoc Med Bras. 2006; 52(4):214-21.

BACHION, MM; PONTIERI, FM. Crenças de pacientes diabéticos acerca da terapia nutricional e sua influência na adesão do tratamento. Ciência e Saúde Coletiva. 2010; 15 (1):151-160.

BATISTA, MCR et al. Avaliação Dietética dos Pacientes Detectados Com Hiperglicemia na "Campanha de Detecção de Casos Suspeitos de Diabetes" no Munícipio de Viçosa, MG. Arquivo Brasileiro de Endocrinologia e Metabologia. Dez 2006; Viçosa, v. 50, n. 6, p. 1041-1049.

BENEDETTI, TRB; MEURER, ST; MORINI, S. Índices antropométricos relacionados a doenças cardiovasculares e metabólicos em idosos. Rev. Educ. Fís/UEM. 1. trim. 2012; v. 23, n. 1, p. 123130.

BRASIL. ANVISA. Agência Nacional de Vigilância Sanitária. Portaria $n^{\circ}$ 29/1998 Disponível em: http://portal.anvisa.gov.br/wps/wcm/connect/ 2a1d950047458eca97dbd73fbc4c6735/PORT ARIA_29_1998.pdf?MOD=AJPERES.

Acesso em: 31/03/2014.

BRASIL. ANVISA. Agência Nacional de Vigilância Sanitária. Resolução RDC $n^{\circ}$ 54/2012 - Disponível em: http://portal.anvisa.gov.br/wps/wcm/connect/ 630a98804d7065b981f1e1c116238c3b/Resol ucao+RDC+n.+54_2012.pdf?MOD=AJPERE S. Acesso em: 02/04/2014.

BRASIL. Ministério da Saúde. Secretaria de Atenção à Saúde. Departamento de Atenção
Básica. Protocolos do Sistema de Vigilância Alimentar e Nutricional - SISVAN na assistência à saúde. Ministério da Saúde, Secretaria de Atenção à Saúde. Departamento de Atenção Básica. Brasília, DF; 2008. 61p. (Série B. Textos Básicos de Saúde)

DEGÁSPARI, CH; MOTTIN, F; RORATO, F. Avaliação do nível de conhecimento de consumidores de produtos diet e light que frequentam um supermercado em Curitiba. Universidade Federal do Paraná. Curitiba-PR. 2006.

FERREIRA, CLRA; FERREIRA, MG. Características Epidemiológicas de pacientes diabéticos da rede pública de saúde - análise a partir do sistema HiperDia. Arq Bras Endocrinol Metab. 2009; 53(1):80-86

HAUN, D R; LESSA, I; PITANGA, FJG. Razão Cintura/Estatura comparado a outros indicadores antropométricos de obesidade como preditor de risco coronariano elevado. Rev Assoc Med Bras. 2009; 55(6): 705-11.

LIPSCHITZ, DA. Screening for nutritional status in the elderly. Primary Care. 1994; 21(1): 55-67.

OLIVEIRA, PB; FRANCO, LJ. Consumo de adoçantes e produtos dietéticos por indivíduos com Diabetes Melito tipo 2, atendidos pelo Sistema Único de Saúde em Ribeirão Preto, SP. Arq Bras Endocrinol Metab. 2010; 54(5):455-42.

PITANGA, FJG. Antropometria na avaliação da obesidade abdominal e risco coronariano. Rev Bras Cineantropom Desempenho Hum. 2011; 13(3):238-241.

SOUSA, TF; NAHAS, MV; SILVA, DAS; DUCA, GFD; PERES, MA. Fatores associados à obesidade central em adultos de Florianópolis, Santa Catarina: estudo de base populacional. Rev Bras Epidemiol. 2011; 14(2): 296-309. 
VIGGIANO, CE. O produto dietético no Brasil e sua importância para indivíduos diabéticos. Centro Universitário Municipal de São Caetano do Sul. São Caetano do Sul, 2003.

ZANINI, RV; ARAÚJO, CL; MARTINEZMESA, J. Utilização de adoçantes dietéticos entre adultos em Pelotas, Rio Grande do Sul, Brasil: um estudo de base populacional. Cad.
Saúde Pública. Mai 2011; Rio de Janeiro, 27(5):924-934.

WHO. WORLD HEALTH ORGANIZATION. Obesity: preventing and managing the global epidemic. Report of a World Health Organization Consultation. Geneva: World Health Organization, 2000. p.256. WHO Obesity Technical Report Series,n.284. 